\title{
Research Paper \\ Comparing Relative Contrast in Three-dimensional Double-echo Steady State With Routine Sequences for a Better Diagnosis of Knee Cartilage Anomalies
}

\section{Sepehr Lotfi Marangaloo ${ }^{1}\left(\mathbb{C}\right.$, Amir Shahriar Ariamanesh $^{2}$, Behzad Aminzadeh ${ }^{3}$, Hormoz Abedi $^{1}$, Alireza Montazerabadi ${ }^{1 *}$ (1)}

1. Medical Physics Research Center, Mashhad University of Medical Sciences, Mashhad, Iran.

2. Department of Orthopedics, School of Medicine, Mashhad University of Medical Sciences, Mashhad, Iran.

3. Department of Radiology, Faculty of Medicine, Mashhad University of Medical Sciences, Mashhad, Iran

\begin{tabular}{|c|c|}
\hline $\begin{array}{l}\text { Use your device toscan } \\
\text { and read the article online }\end{array}$ & Citation Lotfi Marangaloo S, Ariamanesh A Sh, Aminzadeh B, Abedi H, Montazerabadi A. [Comparing Relative Contrast in \\
\hline 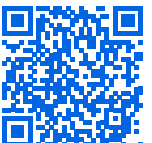 & $\begin{array}{l}\text { Three-dimensional Double-echo Steady State With Routine Sequences for a Better Diagnosis of Knee Cartilage Anomalies (Per- } \\
\text { sian)]. Quarterly of "The Horizon of Medical Sciences". 2020; 26(2):108-117. https://doi.org/10.32598/hms.26.2.2008.1 } \\
\text { dol'https://doi.org/10.32598/hms.26.2.2008.1 }\end{array}$ \\
\hline
\end{tabular}

\section{(i) (8)}

Received: 02 Jul 2019

Accepted: 22 Dec 2019 Available Online: 01 Apr 2020

Key words:

MRI, Osteoarthritis, DESS protocol, STIR, PD

\section{A B STRACT}

Aims This study compared relative contrast values in three-dimensional Double Echo Steady State (DESS) sequences with two flip angles of 40 and 90 degrees and other routine sequences. The obtained data could help to prove the effects of this sequence, compared to the other routine sequences; accordingly, it could be applied for better detection of lesions resulting from cartilage abnormalities.

Methods \& Materials Total, 15 patients, including 9 men (14-56 y) and 6 women (36-65y) with knee pain symptoms were referred to us by an orthopedic physician. All of the investigated patients underwent Magnetic Resonance Imaging (MRI) using DESS sequences with two flip angles of 40 and 90 degrees, as well as STIR , PD, T2, and 3D FLASH. We analyzed the acquired images using the Radiant DICOM Viewer4.6.9 software. We calculated relative contrast values for the selected SAGITAL images per sequence for each patient. The obtained data were analyzed using SPSS.

Findings Friedman and Wilcoxon statistical tests data revealed significant $P$ values $(<0.05)$ for all the sequences examined in this study. The DESS sequence with a flip angle of $90^{\circ}$ presented the most relative contrast value with the average score of 77.2667. The FLASH sequence provided the worst result of 3.733 for the relative contrast value.

Conclusion The DESS sequence with $90^{\circ}$ flip angle indicated the best relative contrast values and provided the best possible signal from the synovial fluid and the cartilage; therefore, it leads to the better examination of articular cartilage in terms of lesion. Accordingly, the DESS sequence with a flip angle of $90^{\circ}$ was the optimal sequence to evaluate cartilage abnormalities among the examined sequences in this study.

\section{Extended Abstract}

\section{Introduction}

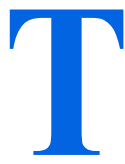

wo-dimensional spin-echo sequences are commonly used to study knee osteoarthritis. The Three-dimensional Double-echo Steady State (3D-DESS) imaging sequence is among the sequences of Siemens Magnetic Resonance Imaging (MRI) machine. This sequence helps to diagnose lesions in knee cartilage by increasing the contrast difference between synovial joint fluid and cartilage. No comprehensive comparison has been made between the DESS sequence and other routine sequences to demonstrate the effectiveness of this sequence.

\section{* Corresponding Author:}

Alireza Montazerabadi, PhD.

Address: Medical Physics Research Center, Mashhad University of Medical Sciences, Mashhad, Iran.

Tel: +98 (915) 1587761

E-mail: alireza.montazerabadi@gmail.com 
Moriya et al. investigated the increase in flip angle from 10 to 90 degrees on Contrast-to-Noise Ratio (CNR). The CNR obtained for the 90-degree DESS sequence had the highest value [1].

Schaefer et al. compared the three DESS, PD FS FSE, and PD SPACE sequences in detecting cartilage anomalies on the human body patella. The DESS sequence provided better relative contrast values, compared to the other sequences [2].

In 1999, Mosher and Pruett conducted a comparative study between the FLASH and DESS sequences on patella cartilage phantom in an albumin solution to assess the diagnosis of cartilage lesions. The DESS sequence performed more effectively in assessing cartilage surface lesions.

We aimed to explore the effectiveness of the DESS sequence to better diagnose osteoarthritis-induced cartilage anomalies in the knee.

\section{Materials and Methods}

This study was performed on 15 patients suspected of having osteoarthritis at Shahid Kamyab and Imam Reza (AS) Hospitals of Mashhad University of Medical Sciences from April 2018 to March 2019.

Considering $\mathrm{s}^{\wedge} 2=30.25$ and $\overline{\mathrm{x}}=18.2$ according to the previous similar article of Han et al. [21]; $d=.2 \bar{x}$ and with the type I error of 0.05 ; the test power of $80 \%$, and the $20 \%$ sample dropouts, the sample size was estimated to be 15 individuals.

A 1.5 T Siemens MRI machine (Avanto series, made in Germany) was used to obtain the required images. Quantitative image processing was performed using Radiant DICOM Viewer 4.6.9 software. Then, using SPSS, the obtained numerical values were compared. To perform statistical analy- sis, the Friedman test and Wilcoxon signed-rank test were employed. $\mathrm{P}<0.05$ was considered statistically significant.

\section{Results}

The Friedman test data for the measured values of relative contrast in the weight-bearing area indicated a significant relationship between the investigated sequences. According to the statistical tests performed for the sequences as well as the measured values of relative contrast for all sequences, the DESS sequence with a flip angle of $90^{\circ}$ and an average value of 77.2667 has the best numerical value of relative contrast among the sequences. Besides, the FLASH sequence has the lowest value in this regard.

\section{Conclusion}

Moria et al. qualitatively compared the DESS sequence at the flip angles of $90^{\circ}$ and $40^{\circ}$. Accordingly, the DESS sequence with a flip angle of $90^{\circ}$ (due to better contrast difference between liquid and cartilage) led to more effective diagnosis of the lesion, compared to the DESS sequence with a flip angle of $40^{\circ}$. The quantitative results obtained by measuring the relative contrast in the present study agree with their findings.

The DESS sequence has significant capabilities in imaging cartilaginous lesions; thus, it is recommended to use this imaging sequence as a complementary sequence along with other routine sequences in medical imaging centers.

\section{Conclusion}

Due to the cooperation of all medical departments, there were no restrictions for performing this study. The DESS sequence is highly efficient for imaging knee cartilage, as well as knee joint fluid. Furthermore, it could improve the

Table 1. The scanning parameters used in this study

\begin{tabular}{|c|c|c|c|c|c|c|c|c|}
\hline $\begin{array}{c}\text { Scanning param- } \\
\text { eters } \\
\text { Sequences }\end{array}$ & TR (ms) & TE (ms) & $\begin{array}{l}\text { Cross- } \\
\text { section } \\
\text { Thickness } \\
(\mathrm{mm})\end{array}$ & $\begin{array}{l}\text { Number of } \\
\text { Slides }\end{array}$ & $\begin{array}{l}\text { FOV } \\
\text { For Frequency } \\
\text { Coding }\end{array}$ & $\begin{array}{l}\text { FOV } \\
\text { For Phase } \\
\text { Coding }\end{array}$ & $\begin{array}{c}\text { Scan Time } \\
\text { (Second) }\end{array}$ & $\begin{array}{c}\text { NEX } \\
\text { (Number } \\
\text { of Scans) }\end{array}$ \\
\hline DESS90 & 21.36 & 7.57 & 1.5 & 64 & 180 & 100 & $6: 30$ & 1 \\
\hline DESS40 & 21.36 & 7.57 & 1.5 & 64 & 180 & 100 & $6: 44$ & 1 \\
\hline FLASH & 10 & 4.92 & 1.5 & 64 & 180 & 100 & 4:09 & 1 \\
\hline STIR & 3700 & 40 & 3 & 32 & 180 & 100 & $2: 44$ & 1 \\
\hline PD & 2400 & 41 & 3 & 32 & 180 & 100 & $3: 55$ & 1 \\
\hline T2W FSE & 3740 & 80 & 3 & 32 & 180 & 100 & $2: 27$ & 1 \\
\hline
\end{tabular}


contrast ratio of joint fluid to cartilage; thus, it could help to better diagnose knee cartilage lesions.

\section{Ethical Considerations}

Compliance with ethical guidelines

This research project obtained code of ethics with the ID of IR.MUMS.MEDICAL.REC.1397.215, which was approved on 23.05.2018.

Funding

The financial resources of this project have been provided by the Research Center of Mashhad University of Medical Sciences and have been registered in the Pajouhan system (Code: 961866).

\section{Authors' contributions}

Conceptualization: Alireza montazerabadi, Amir shahriyar ariamanesh; Methodology: Behzad Aminzade; Investigation :Hormoz Abed, \& Sepehr Lotfi; writing_review and editing: Sepehr Lotfi; Analysis: Alireza Montazerabadi, Sepehr Lotfi.

\section{Conflicts of interest}

There is no conflict of interest to be declared by the authors.

\section{Acknowledgements}

We are very grateful to Mashhad University of Medical Sciences Research Center as the funding provider of this research; Mashhad Medical Physics Department, Medical Imaging Department of Shahid Kamyab Hospital of Mashhad University of Medical Sciences, Orthopedic Department of Imam Reza Hospital of Mashhad University of Medical Sciences, and the esteemed board that guided us in carrying out this research. Eventually, we would like to thank the esteemed officials of Ofogh-e-Danesh ScientificResearch Journal, who helped us in developing this article in the best possible manner by providing us their best guidance. 


\title{
بهبود كنتراست نسبى در سكانس تصويربردارى سلبعدى DESS به منظور تشخيص بهتر

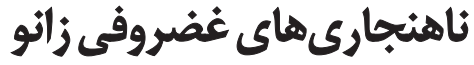

\author{
سيهر لطفى مرنكلو' ه، امير شهريار آريامنش'، بهزاد امينزاده'، هرمز عابدى'، "عليرضا منتظرابدى' \\ 1. ا.مركز تحقيقات فيزيك يزشكى، دانشعاه علوميزشكى مشهد، مشهيد، يران.

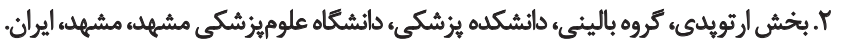

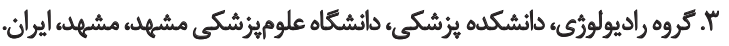

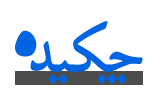

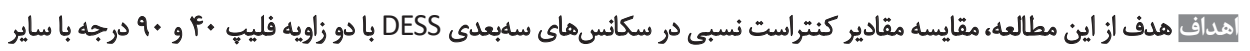

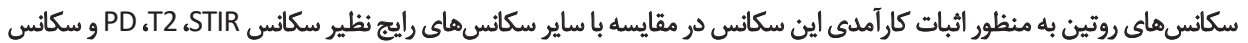

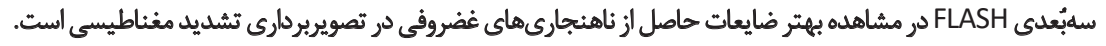

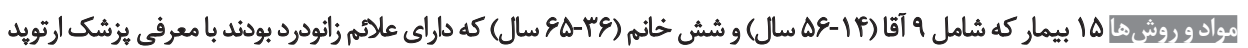

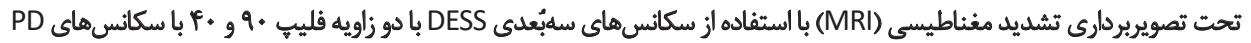

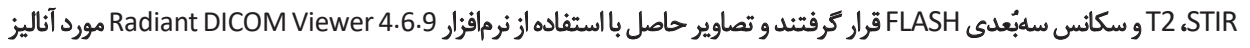

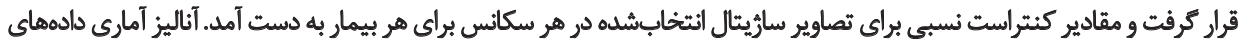

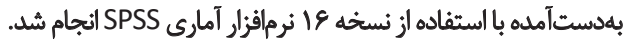

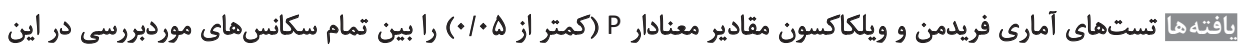

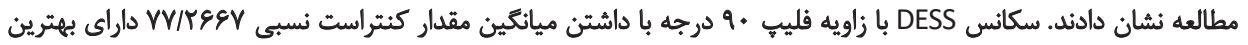

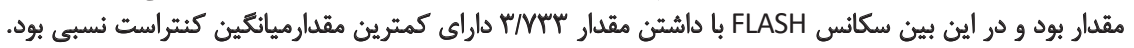

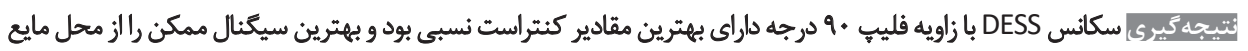

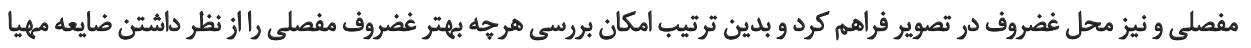

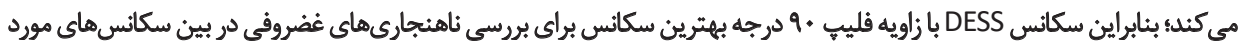

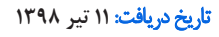

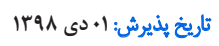

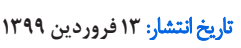

مطالعه روى استئوآرتريت در زانو به منظور تشخيص زودهنكام

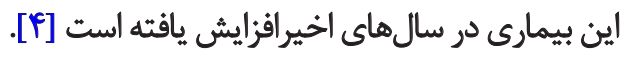

مقدمه

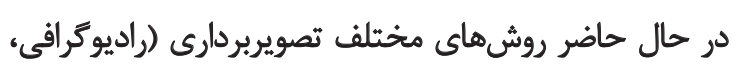

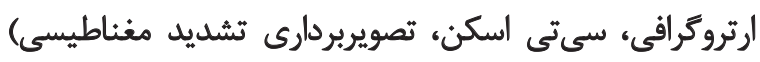

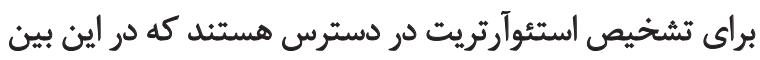

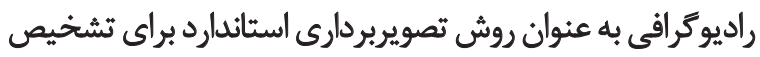

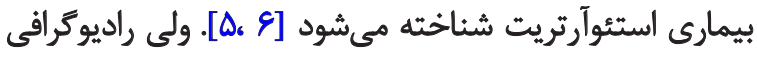

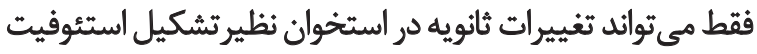

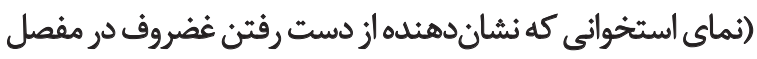

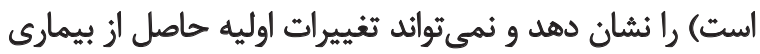

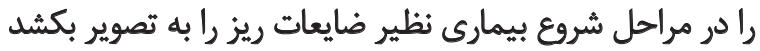

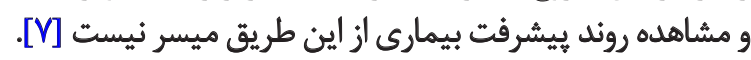

بيمارى استئوآرتريت يك بيمارى مفصلى مزمن است كه با

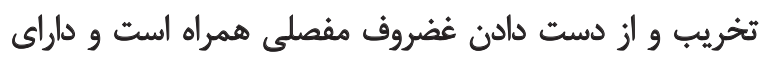

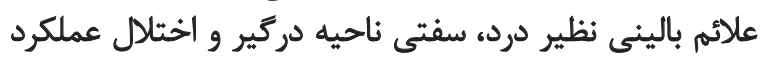

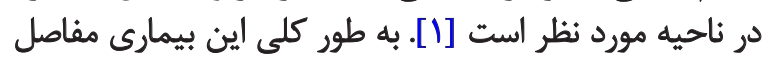

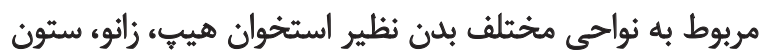

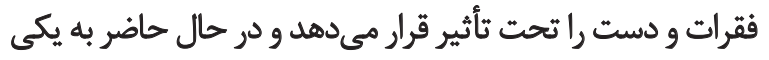

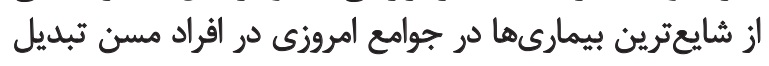

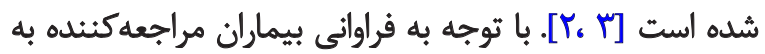

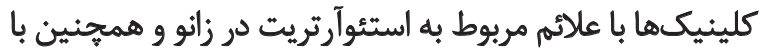

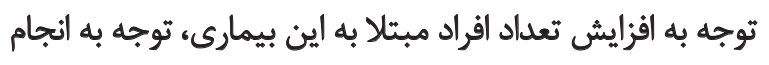


DESS و ساير سكانسهاى روتين در جهت اثبات كارآمدى اين

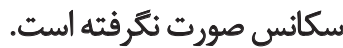

به منظور تشخيص بهتر ضايعات غضروفى و با توجه به ويرثى دئى

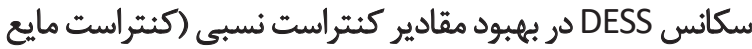

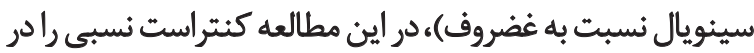

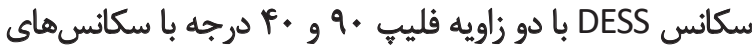

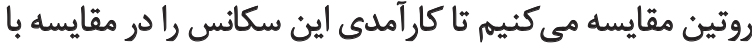

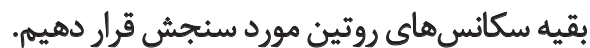

مواد وروشى اثما

در اين مطالعه 10 بيمار كه داراي علائم زانو درد بودند توسط

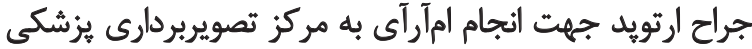

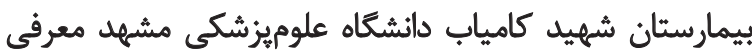

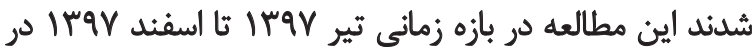

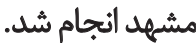
روش نمونه كيرى

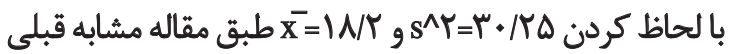

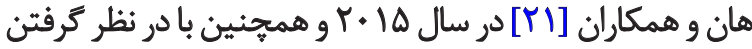
de.rx

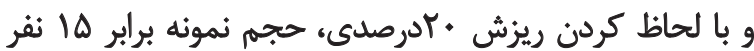
كرفته شد.

\section{روش - انجام كار}

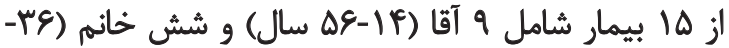

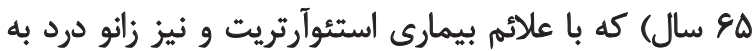

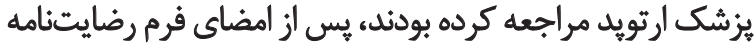

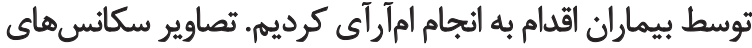

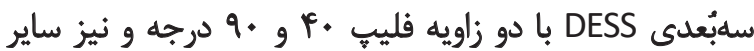

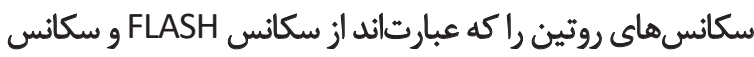

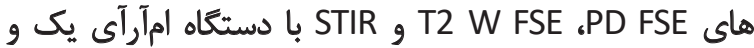
نيم تسلا زيمنس (سرى آوانتو ساخت كشور آلمان) از بيماران

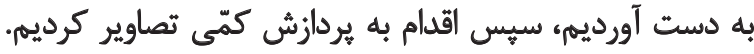

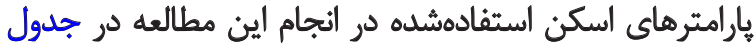

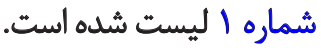

Radiant DICOM يردازش كتمى تصاوير با استفاده از نرمافزار

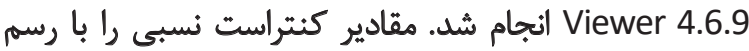

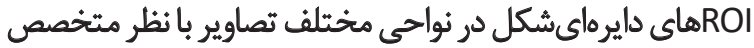

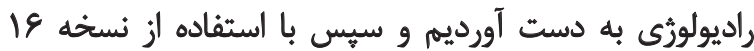

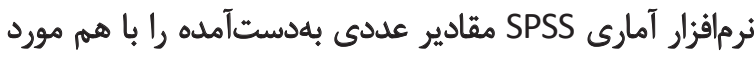

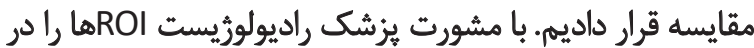

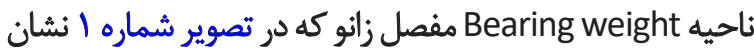

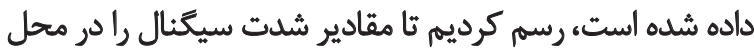

تصويربردارى تشديد مغناطيسى يك روش غيرتهاجمي براى بـ بافي

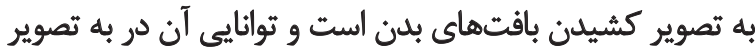
كشيدن بافت نرم با كنتراست عالى بالى باعث شده است است تا اين روش

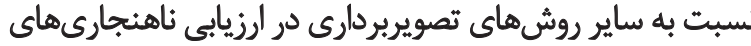

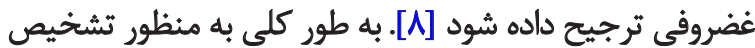

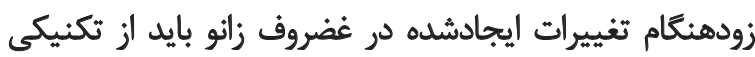

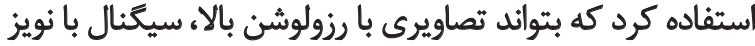

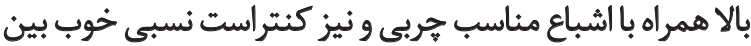

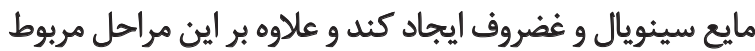

به يردازش تصوير نيز در زمان قابل قبولى انجام شود [ـ 19 19]. در حال حاضر به طور متداول براى انجام مطالعه روى

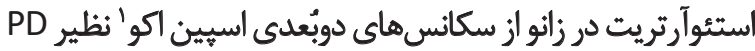

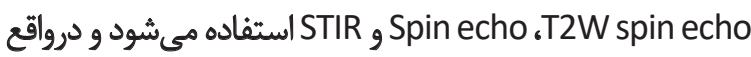
سكانسهاى دوبُعدى اسيّين اكو جزء سكانسهاى توصيهشده

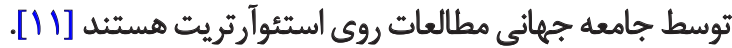
سكانسهاى سلبُعدى داراى مزاياى زيادى هستند، از جمله

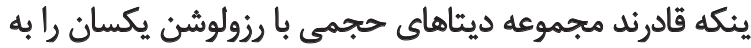

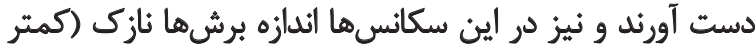

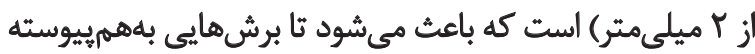

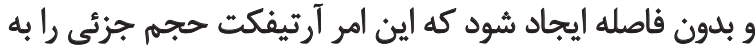

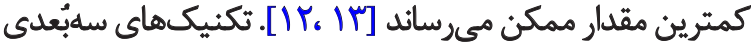

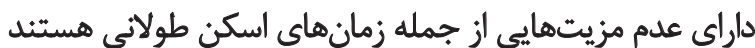

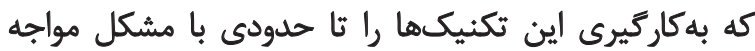

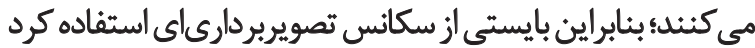

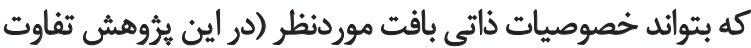

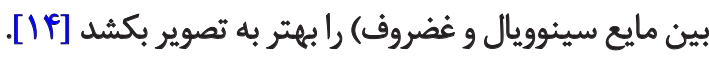

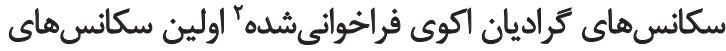

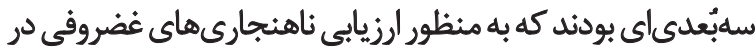

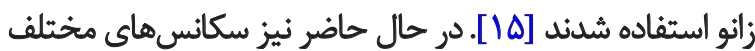

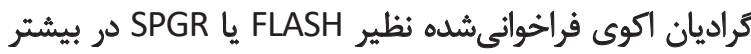

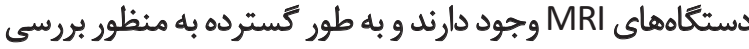

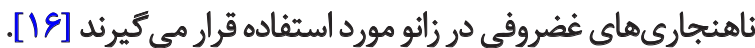
سكانس تصويربردارى 3D DESS" يكى از سكانس هاى دستَّاه

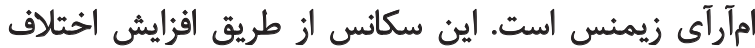

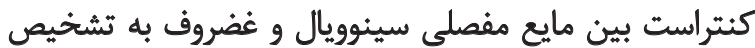

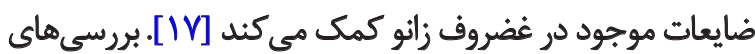

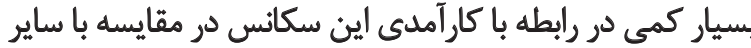

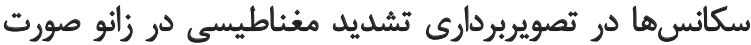

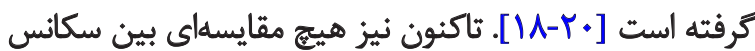

1. Spin echo

2. Gradient Echo (GRE)

3. 3 Dimensional double echo steady state 
كزارش شده است. مقايسه ميانكَين رتبهها كه در جدول شماره

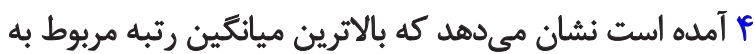

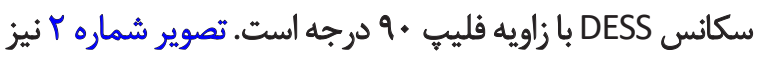

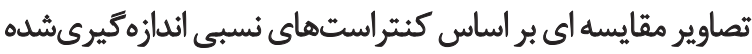
براى هر سكانس را نشان مى دهد.

ث

هدف از اين مطالعه بررسى كار آمدى سكانس DESS در مقايسه با ساير سكانسهاى روتين به منظور تشخيص بهارئ بهري

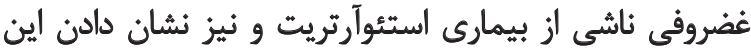

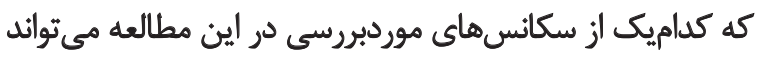

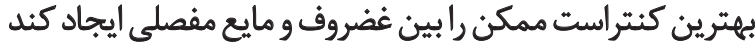

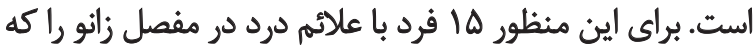

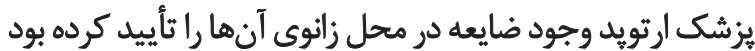

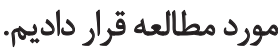

اندازهيرى مقادير كنتراست نسبى در اين مطالعه براى هركدام

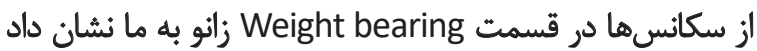

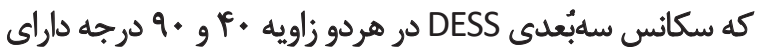

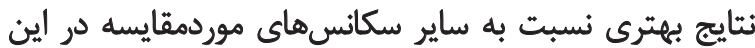

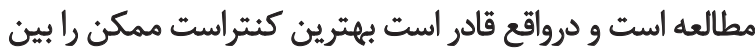

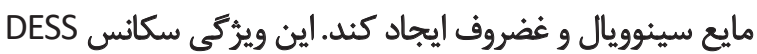

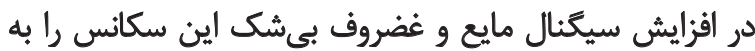

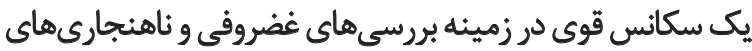

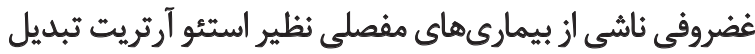

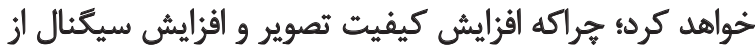

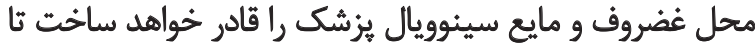
ديد بهترى از محل ضايعه داشته باشد و بنابر براين به به تشخيص بهتر

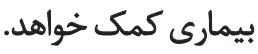

مايع سينويال (S1) و غضروف (SI2) به دست آوريم و با استفاده

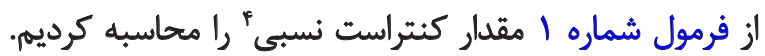

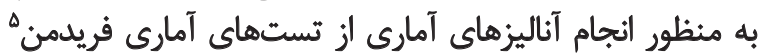

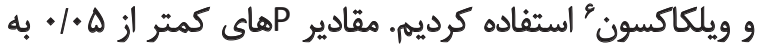
عنوان مقادير معنادار آمارى در نظر كردفته شد. مقادير

\section{Relative Contrast $=\frac{S / 1-S / 2}{S / 1+S / 2} \times 100$}

يافتهها

اجراى تست آمارى فريدمن براى مقادير اندازهكيرىشده

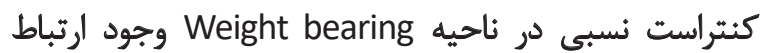

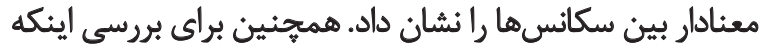

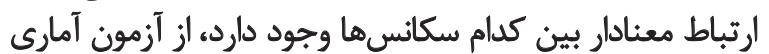

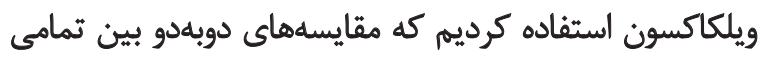

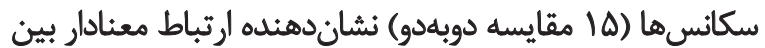

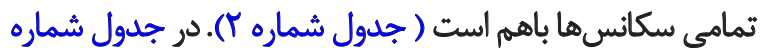

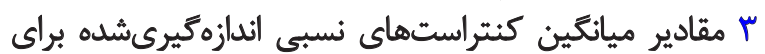

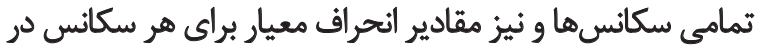

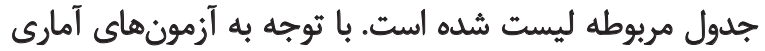

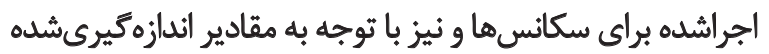

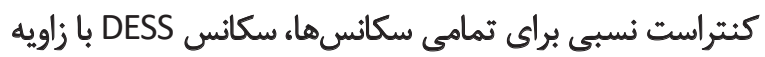

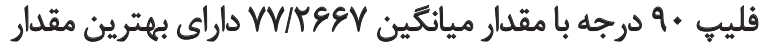

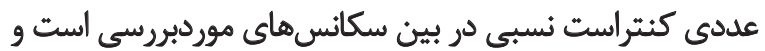

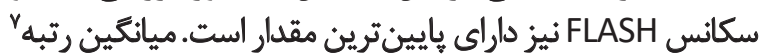

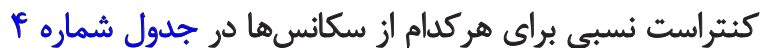

\footnotetext{
4. Relative contrast

5. FRIEDMAN test

6. Wilcoxon signed ranks

7. Mean rank
}

جدول ا. يارامترهاي اسكن استفادهشده در اين مطالعه

\begin{tabular}{|c|c|c|c|c|c|c|c|c|}
\hline $\begin{array}{c}\text { NEX } \\
\text { (تعداد دفعات) }\end{array}$ & زمان اسكن) & 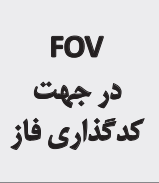 & 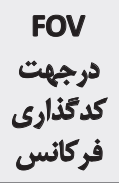 & اسلايسهـا & 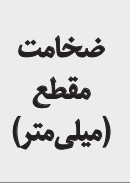 & (TE) ms & (TR) ms & سيار المترهاي \\
\hline 1 & q:" & $1 .$. & $M$. & gt & $1 / 0$ & $V_{j} \Delta V$ & Mi,re & DES\$90 \\
\hline 1 & s:tf & 1.0 & M. & gr & $1 / \Delta$ & $V_{j} \Delta \mathrm{V}$ & m, & DESS40 \\
\hline 1 & $f:-q$ & $1+\infty$ & $M$ & gf & $1 / 0$ & fiqr & 1. & FLASH \\
\hline 1 & T:FF & $1 .$. & $\mathrm{M}$. & $r$ & $r$ & r. & $r .$. & STIR \\
\hline 1 & $r \backsim \Delta$ & 1.0 & M. & MT & $r$ & il & re.. & PD \\
\hline 1 & Y:TY & 1.0 & M. & rr & $r$ & A. & me. & T2W FSE \\
\hline
\end{tabular}




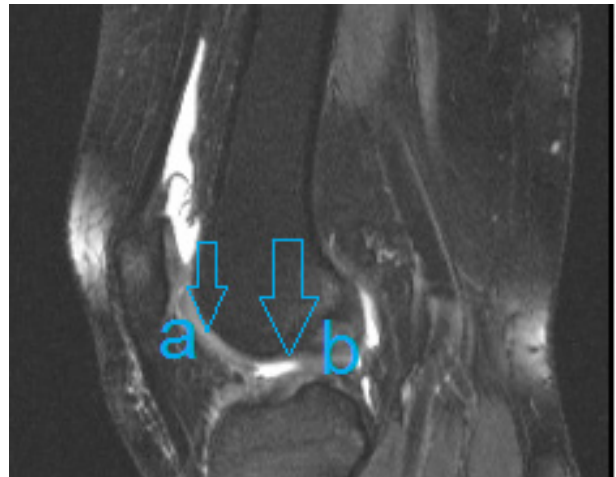

A

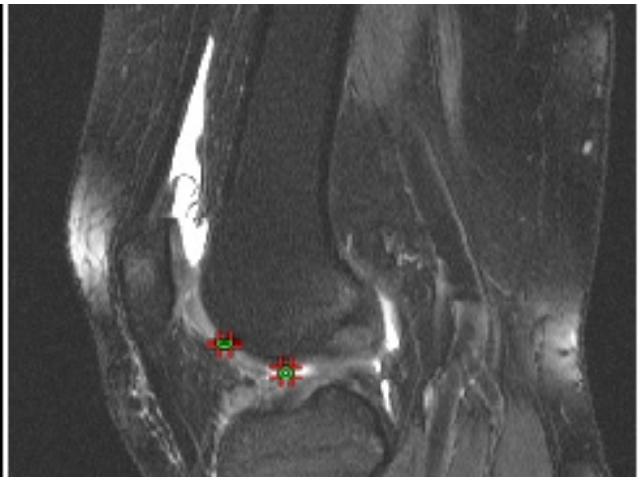

B

أنّانت

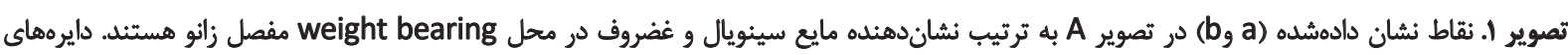

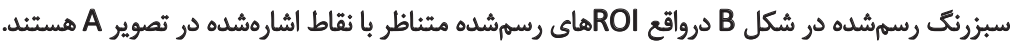
جدول r. آزمون ويلكاكسون و مقايسهاى دوبهدوى سكانسها باهم

\begin{tabular}{|c|c|c|c|c|c|c|c|c|c|c|c|c|c|c|c|}
\hline $\begin{array}{l}0 \\
\frac{1}{1} \\
\frac{1}{1}\end{array}$ & $\begin{array}{l}\stackrel{\varrho}{\underline{E}} \\
\stackrel{\Delta}{F}\end{array}$ & $\begin{array}{l}\underline{\underline{E}} \\
\dot{b} \\
\text { d }\end{array}$ & $\frac{\bar{T}}{\frac{5}{4}}$ & $\frac{\frac{1}{y}}{\frac{1}{4}}$ & 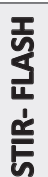 & $\begin{array}{l}\text { 오․ } \\
\text { 岃 } \\
\text { ㅅ․ }\end{array}$ & $\begin{array}{l}\text { 워․ } \\
\text { 㟔 } \\
\text { 定 }\end{array}$ & 占曷莒 & 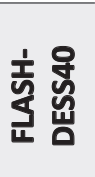 & 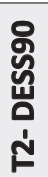 & 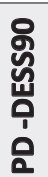 & 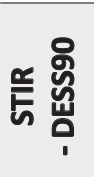 & 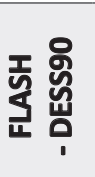 & 㝵总 & مثايسه شائسداي \\
\hline
\end{tabular}

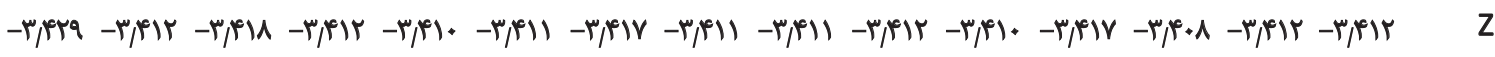

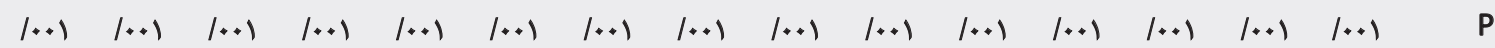
إنّان

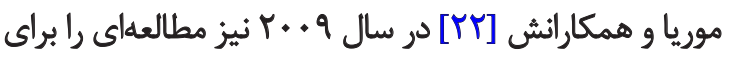

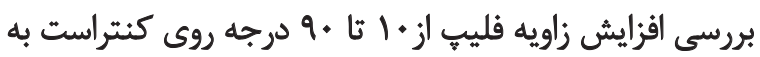

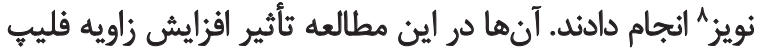

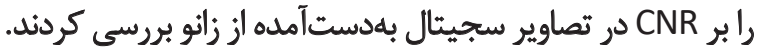

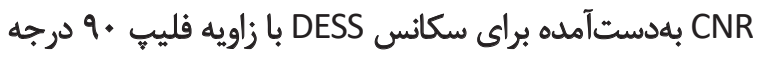

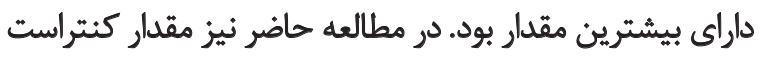

در مطالعه قبلى انجامشده [19] در اين زمينه كه به مقايسه

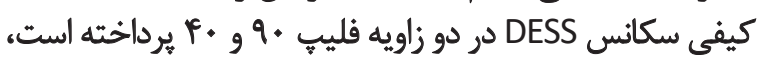

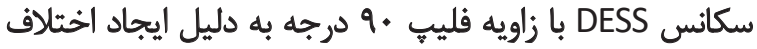

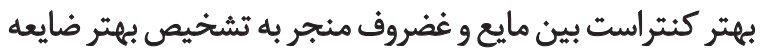

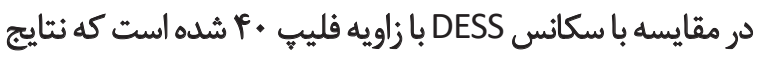

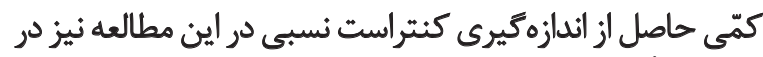
توافق و ثأييد با آن است.

8. Contrast to Noise Ratio (CNR) 

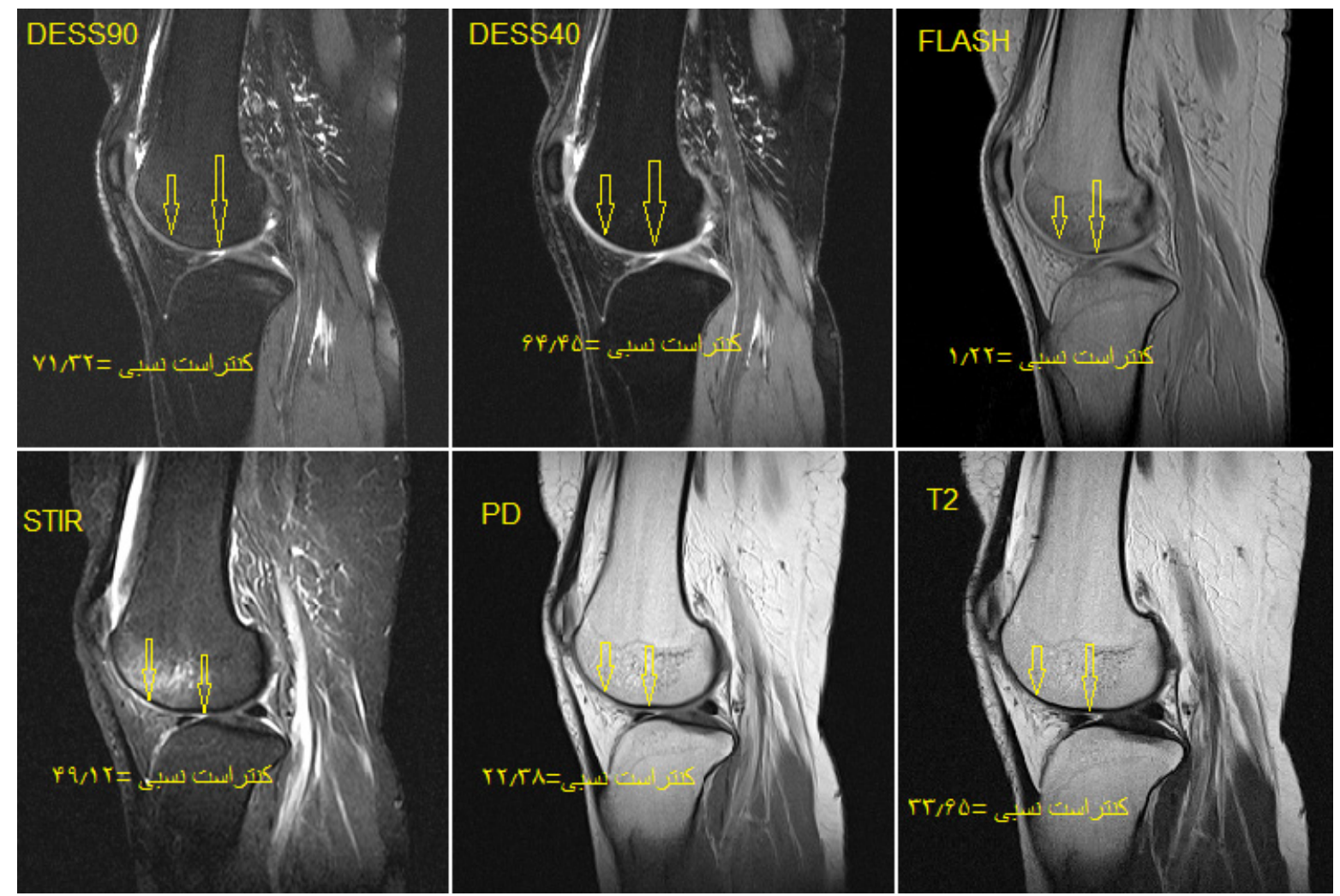

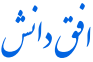

تصوير T. تصوير زانوى يك بيمار خانم بآساله با علاثم درد در زانو. مقادير كنتراست نسبى بين دو نقطه اشارمشده توسط فلش اندازمكيرى شده است كه نشان

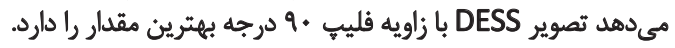

تشخيص ناهنجارىهاى غضروفى روى يتلاى جسد انسان انجام

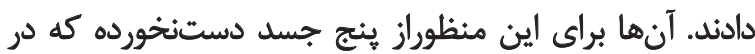

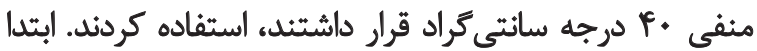

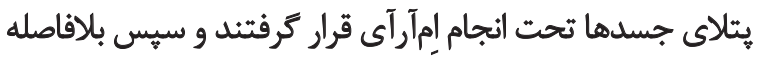

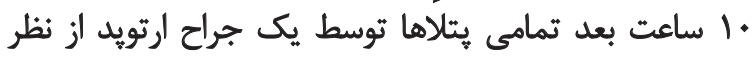

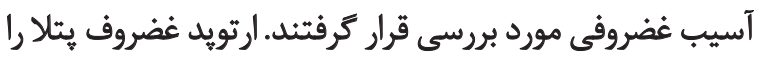

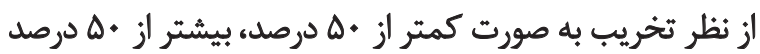

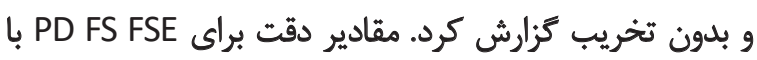

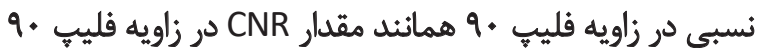

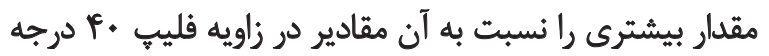

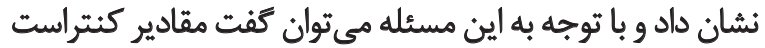

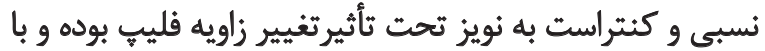
افزايش آن رابطه مستقيم دارند.

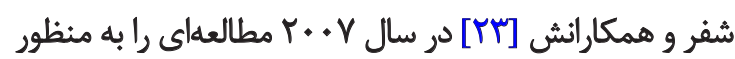

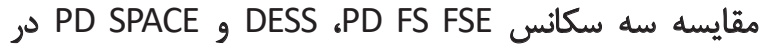

جدول F . ميانكين رتبهاي كنتراست نسبى براى سكانس ها.

\begin{tabular}{|c|c|}
\hline ميانغينين رتبه & سكانسهاى موردبر رسى \\
\hline$\varepsilon_{1 .}$ & DESS90 \\
\hline$\Delta /$. & DESS40 \\
\hline$r_{1 .}$. & STIR \\
\hline$r_{1 .+}$ & $\mathrm{T} 2$ \\
\hline$r / .+$ & PD \\
\hline $1, \ldots$ & FLASH \\
\hline
\end{tabular}

أن 
منابع مالى اين طرح يروهشى توسط مركز تحقيقات دانشكاه

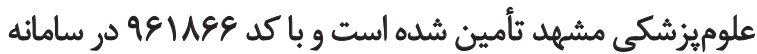

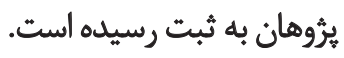

\section{مشاركت نويسند مكان}

مفهوم سازى: عليرضا منتظرآبادى ، اميرشهريار آريامانش؛ زإن

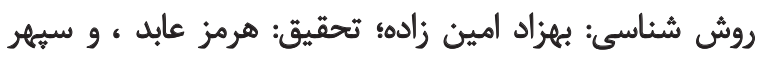
لطفى؛ نوشتن - بررسى و ويرايش: سيهر إدير لطفي؛ تحليل: عليرضا منتظر آبادى ، سيهر لطفى بروسي

$$
\text { ت تعارض مثاقع }
$$

بنابر اظهار نويسندكان هيج تعارض منافعى وجود ندارد.

$$
\text { تشكر وقدروانى }
$$

از مركز تحقيقات دانشعاه علوميزشكى مشهيد به خاطر تأمين

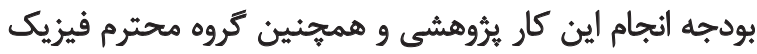

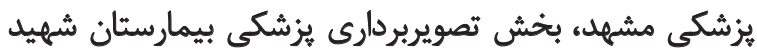

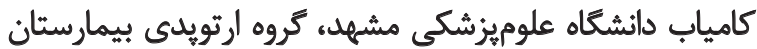

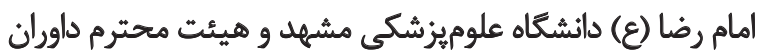

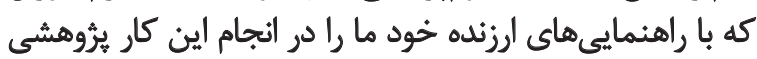

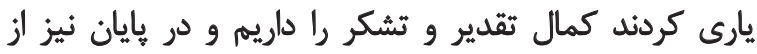

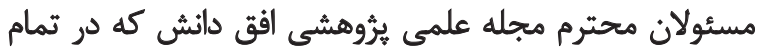

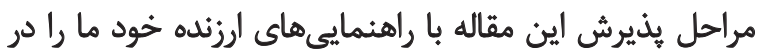

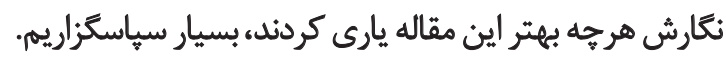

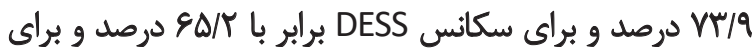

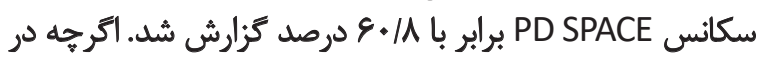

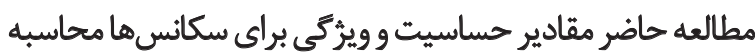

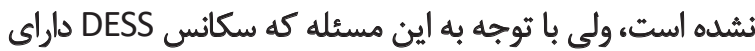

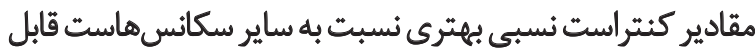

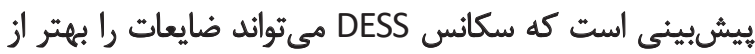
ساير سكانس هاى موردبررسي در اين مطالعه به تصوير بكشد.

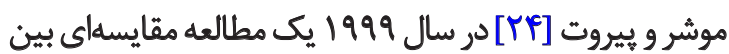

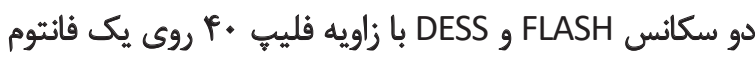

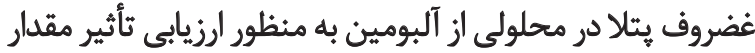

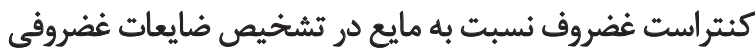

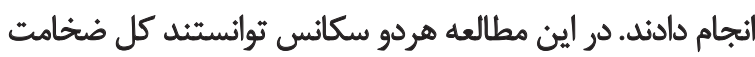

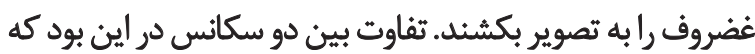

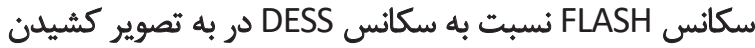

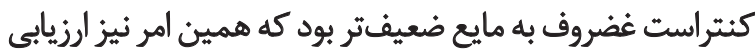

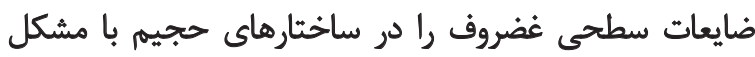

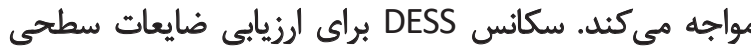

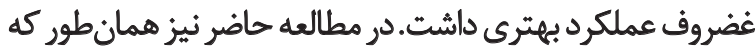

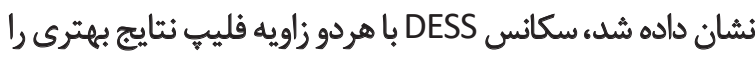

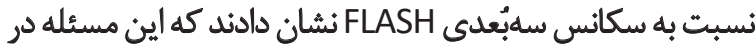
تأييد و توافق نتايج حاصل از مطالعه موشر و بيروت است.

\section{نتيجلمَيرى}

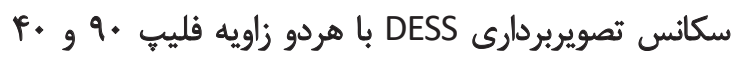

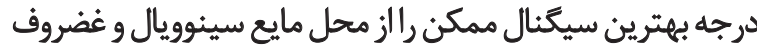

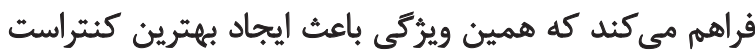

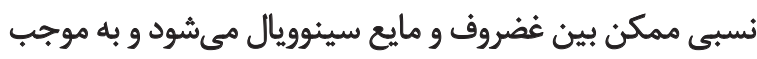

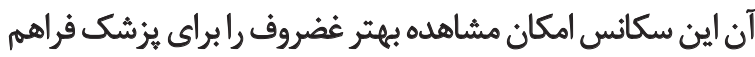

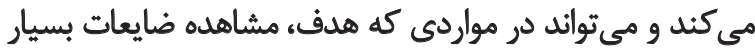

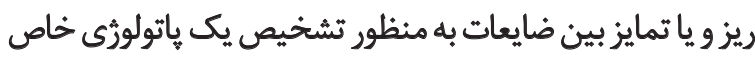

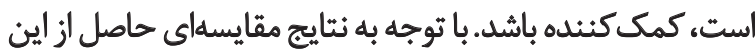

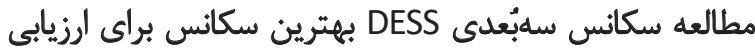

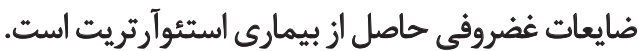

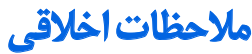

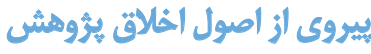

IR.MUMS. اين طرح يُروهشى داراى كد اخلاق با شناسه

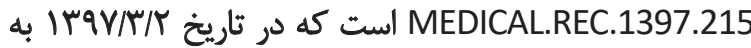




\section{References}

[1] Shapiro LM, McWalter EJ, Son MS, Levenston M, Hargreaves BA, Gold GE. Mechanisms of osteoarthritis in the knee: MR imaging appearance. Journal of Magnetic Resonance Imaging. 2014; 39(6):1346-56. [DOI:10.1002/jmri.24562] [PMID] [PMCID]

[2] Wildi LM, Martel-Pelletier J, Abram F, Moser T, Raynauld JP, Pelletier JP. Assessment of cartilage changes over time in knee osteoarthritis disease-modifying osteoarthritis drug trials using semiquantitative and quantitative methods: Pros and cons. Arthritis Care \& Research. 2013; 65(5):686-94. [DOI:10.1002/acr.21890] [PMID]

[3] Sharma L. Osteoarthritis year in review 2015: Clinical. Osteoarthritis and Cartilage. 2016; 24(1):36-48. [DOI:10.1016/j.joca.2015.07.026] [PMID] [PMCID]

[4] March LM, Bachmeier CJM. Economics of osteoarthritis: A global perspective. Baillière's Clinical Rheumatology. 1997; 11(4):817-34. [DOI:10.1016/S0950-3579(97)80011-8]

[5] Coumas JM, Palmer WE. Knee arthrography: Evolution and current status. Radiologic Clinics. 1998; 36(4):703-28. [DOI:10.1016/S00338389(05)70057-9]

[6] Brittberg M, Lindahl A, Nilsson A, Ohlsson C, Isaksson O, Peterson L. Treatment of deep cartilage defects in the knee with autologous chondrocyte transplantation. The New England Journal of Medicine. 1994; 331(14):889-95. [DOI:10.1056/NEJM199410063311401] [PMID]

[7] Jones G, Ding C, Scott F, Glisson M, Cicuttini F. Early radiographic osteoarthritis is associated with substantial changes in cartilage volume and tibial bone surface area in both males and females. Osteoarthritis and Cartilage. 2004; 12(2):169-74. [DOI:10.1016/j.joca.2003.08.010] [PMID]

[8] Van Dyck P, Vanhevel F, Vanhoenacker FM, Wouters K, Grodzki DM, Gielen JL, et al. Morphological MR imaging of the articular cartilage of the knee at 3 T-comparison of standard and novel 3D sequences. Insights into Imaging. 2015; 6(3):285-93. [DOI:10.1007/s13244-015-0405-1] [PMID] [PMCID]

[9] Peterfy CG, Gold G, Eckstein F, Cicuttini F, Dardzinski B, Stevens R. MRI protocols for whole-organ assessment of the knee in osteoarthritis. Osteoarthritis and Cartilage. 2006; 14 Suppl A:A95-111. [DOI:10.1016/j. joca.2006.02.029] [PMID]

[10] Siepmann DB, McGovern J, Brittain JH, Reeder SB. High-resolution 3D cartilage imaging with IDEAL SPGR at 3 T. American Journal of Roentgenology. 2007; 189(6):1510-5. [DOI:10.2214/AJR.07.2661] [PMID]

[11] Hunter DJ, Guermazi A. Imaging techniques in osteoarthritis. PM \& R: The Journal of Injury, Function, and Rehabilitation. 2012; 4(5 Suppl):S68-74. [DOI:10.1016/j.pmrj.2012.02.004] [PMID]

[12] Milewski MD, Smitaman E, Moukaddam H, Katz LD, Essig DA, Medvecky MJ, et al. Comparison of 3D vs. 2D fast spin echo imaging for evaluation of articular cartilage in the knee on a 3T system scientific research. European Journal of Radiology. 2012; 81(7):1637-43. [DOI:10.1016/j. ejrad.2011.04.072] [PMID]

[13] Crema MD, Nogueira-Barbosa MH, Roemer FW, Marra MD, Niu J, Chagas-Neto FA, et al. Three-dimensional turbo spin-echo Magnetic Resonance Imaging (MRI) and semiquantitative assessment of knee osteoarthritis: Comparison with two-dimensional routine MRI. Osteoarthritis and Cartilage. 2013; 21(3):428-33. [DOI:10.1016/j.joca.2012.12.011] [PMID]

[14] Heron CW, Calvert PT. Three-dimensional gradient-echo MR imaging of the knee: Comparison with arthroscopy in 100 patients. Radiology. 1992; 183(3):839-44. [DOI:10.1148/radiology.183.3.1584944] [PMID]
[15] Kijowski R, Blankenbaker DG, Woods M, Del Rio AM, De Smet AA, Reeder SB. Clinical usefulness of adding 3D cartilage imaging sequences to a routine knee MR protocol. American Journal of Roentgenology. 2011; 196(1):159-67. [DOI:10.2214/AJR.09.4095] [PMID]

[16] Kijowski R, Gold GE. Routine 3D magnetic resonance imaging of joints. Journal of Magnetic Resonance Imaging. 2011; 33(4):758-71. [DOI:10.1002/jmri.22342] [PMID] [PMCID]

[17] Hardy PA, Recht MP, Piraino D, Thomasson D. Optimization of a dual echo in the steady state (DESS) free-precession sequence for imaging cartilage. Journal of Magnetic Resonance Imaging. 1996; 6(2):329-35. [DOI:10.1002/jmri.1880060212] [PMID]

[18] Lavdas E, Topalzikis T, Mavroidis P, Kyriakis I, Roka V, Kostopoulos S, et al. Comparison of PD BLADE with Fat Saturation (FS), PD FS and T2 3D DESS with Water Excitation (WE) in detecting articular knee cartilage defects. Magnetic Resonance Imaging. 2013; 31(8):1255-62. [DOI:10.1016/j.mri.2013.06.007] [PMID]

[19] Moriya S, Miki Y, Kanagaki M, Matsuno Y, Miyati T. $90^{\circ}$-flip-angle threedimensional Double-Echo Steady-State (3D-DESS) magnetic resonance imaging of the knee: Isovoxel cartilage imaging at 3T. European Journal of Radiology. 2014; 83(8):1429-32. [DOI:10.1016/j.ejrad.2014.04.034] [PMID]

[20] Roemer FW, Kwoh CK, Hannon MJ, Crema MD, Moore CE, Jakicic $\mathrm{JM}$, et al. Semiquantitative assessment of focal cartilage damage at 3T MRI: A comparative study of dual echo at steady state (DESS) and Intermediate-Weighted (IW) fat suppressed fast spin echo sequences. European Journal of Radiology. 2011; 80(2):e126-31. [DOI:10.1016/j. ejrad.2010.07.025] [PMID]

[21] Han CH, Park HJ, Lee SY, Chung EC, Choi SH, Yun JS, et al. IDEAL 3D spoiled gradient echo of the articular cartilage of the knee on 3.0 T MRI: A comparison with conventional 3.0 T fast spin-echo T2 fat saturation image. Acta Radiologica (Stockholm, Sweden: 1987). 2015; 56(12):147986. [DOI:10.1177/0284185114556097] [PMID]

[22] Moriya S, Miki Y, Yokobayashi T, Ishikawa M. Three-dimensional Double-Echo Steady-State (3D-DESS) magnetic resonance imaging of the knee: contrast optimization by adjusting flip angle. Acta Radiologica (Stockholm, Sweden: 1987). 2009; 50(5):507-11. [DOI:10.1080/02841850902849444] [PMID]

[23] Schaefer FK, Kurz B, Schaefer PJ, Fuerst M, Hedderich J, Graessner $\mathrm{J}$, et al. Accuracy and precision in the detection of articular cartilage lesions using magnetic resonance imaging at 1.5 Tesla in an in vitro study with orthopedic and histopathologic correlation. Acta Radiologica (Stockholm, Sweden: 1987). 2007; 48(10):1131-7. [DOI:10.1080/02841850701549583] [PMID]

[24] Mosher TJ, Pruett SW. Magnetic resonance imaging of superficial cartilage lesions: Role of contrast in lesion detection. Journal of Magnetic Resonance Imaging. 1999; 10(2):178-82. [DOI:1002/(SICI)15222586(199908)10:2<178::AID-JMRI11>3.0.CO;2-W] 